\title{
CHP-NY-ESO-1 Peptide Vaccine IMF-001
}

National Cancer Institute

\section{Source}

National Cancer Institute. CHP-NY-ESO-1 Peptide Vaccine IMF-001. NCI Thesaurus. Code C62598.

A peptide cancer vaccine containing nanoparticles of cholesteryl hydrophobized pullulan (CHP) complexed with the cancer-testis antigen NY-ESO-1 protein, with potential immunostimulating and antineoplastic activities. Upon administration, CHP-NY-ESO-1 peptide vaccine IMF-001 may stimulate the host immune system to mount a humoral and cytotoxic T-cell response against tumor cells expressing NY-ESO-1 antigen, resulting in tumor cell lysis. The self-aggregating CHP, composed of a pullulan backbone and cholesterol branches, forms stable colloidal nanoparticles in water. NY-ESO-1, an antigen found in normal testis, is upregulated in various cancers, including bladder, breast, hepatocellular, melanoma, and prostate cancers. 